\title{
Electrode Dimension Effects on Blue-Phase Liquid Crystal Displays
}

\author{
Kuan-Ming Chen, Jin Yan, Shin-Tson Wu, Yu-Pei Chang, Chen-Chu Tsai, and Jyh-Wen Shiu
}

\begin{abstract}
Electrode dimension effects on the electro-optic properties of a polymer-stabilized blue-phase liquid crystal (PS-BPLC) are investigated experimentally. By reducing the width and gap of the in-plane-switching (IPS) electrodes, the operating voltage of the PS-BPLC is decreased significantly while keeping over $80 \%$ transmittance. This unexpected high transmittance results from fringing field migration into the electrode region, which in turn activates more BPLC material and enlarges the aperture ratio. Further reducing electrode dimension to $2 \mu \mathrm{m}$ would cause some undesirable effects, such as increased response time and hysteresis due to electrostriction effect and phase transition, and ultimately damage the polymer networks.
\end{abstract}

Index Terms-BPLC, electrode effects.

\section{INTRODUCTION}

$\mathbf{P}$ OLYMER-STABILIZED blue-phase liquid crystal (PSBPLC) is emerging as next-generation display technology because of its submillisecond response time, no need for alignment layer, isotropic dark state, and insensitivity to cell gap if an in-plane-switching (IPS) cell is employed [1]-[5]. However, some technical challenges such as high operating voltage, hysteresis, residual birefringence, and wide $\mathrm{BP}$ temperature range remain to be overcome before widespread applications can be realized. To reduce voltage, two approaches are commonly practiced: developing large Kerr constant $(K)$ BPLC materials, and exploiting device design for efficiently generating strong and deep penetrating fields [6], [7]. In the material forefront, recently a BPLC with $K \sim 13.7 \mathrm{~nm} / \mathrm{V}^{2}$ has been reported [8]. On the device simulations, small IPS electrode [3], [4], protrusion electrode [6], wall-shaped electrode [9], and corrugated electrode [10] have been proposed. Each approach has its own pros and cons.

In this paper, we experimentally investigate the IPS electrode dimension effects on the electro-optic properties of a large- $\mathrm{K}$ PS-BPLC composite. Our results show that the operating voltage is reduced dramatically by narrowing electrode dimension while the transmittance keeps above $80 \%$, which is higher than what the UCF model predicted [3]. This unexpected high transmittance results from dielectric coupling into the electrode region which in turn enlarges the effective aperture ratio. However, these strong electric fields also cause some undesirable effects such as electrostriction and phase transition, leading to slower response time and wider hysteresis.

Manuscript received March 15, 2011; revised April 28, 2011; accepted May 04, 2011. Date of current version June 07, 2011. The work of K. M. Chen, J. Yan, and S. T. Wu was supported by Industrial Technology Research Institute (Taiwan).

K. M. Chen, J. Yan, and S. T. Wu are with the College of Optics and Photonics, University of Central Florida, Orlando, FL 32816 USA (e-mail: kuanming@creol.ucf.edu, jyan@creol.ucf.edu, swu@mail.ucf.edu).

Y.-P. Chang, C.-C. Tsai, and J.-W. Shiu are with Display Technology Center, Industrial Technology Research Institute, Chutung, Hsinchu 310, Taiwan (e-mail: changyupei@itri.org.tw; bamboo_tsai@itri.org.tw; jwshiu@itri.org.tw).

Color versions of one or more of the figures are available online at http:// ieeexplore.ieee.org.

Digital Object Identifier 10.1109/JDT.2011.2153177

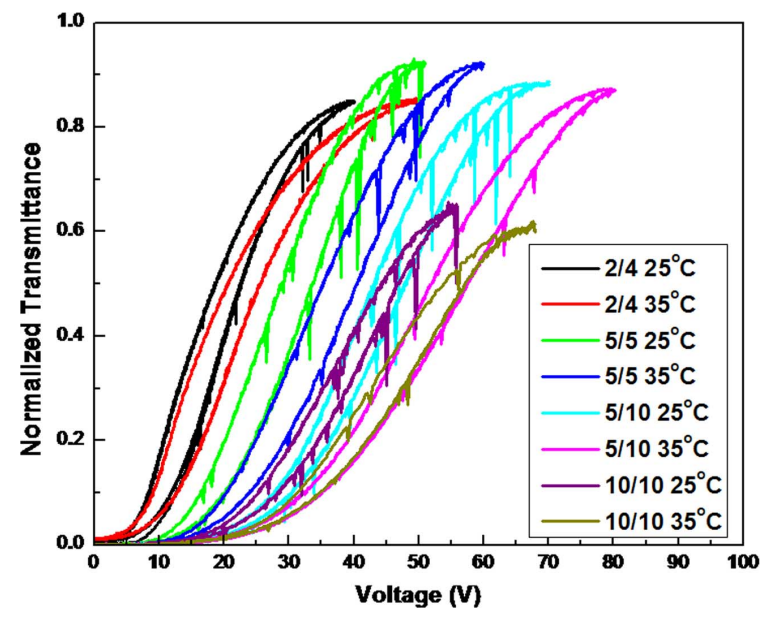

Fig. 1. Measured VT curves of the PS-BPLC samples with different electrode dimensions at $25^{\circ} \mathrm{C}$ and $35^{\circ} \mathrm{C}$. $\lambda=633 \mathrm{~nm}$.

\section{EXPERIMENT}

To reduce operating voltage, in our experiment we used Chisso JC-BP01M BPLC mixture whose birefringence is $\Delta n \sim 0.17$, dielectric anisotropy $\Delta \varepsilon \sim 94$, and Kerr constant $K \sim 13.7 \mathrm{~nm} / \mathrm{V}^{2}$ [8]. To study electrode dimension effects, we prepared five IPS cells in which the bottom glass substrate was coated with a transparent ITO (indium tin oxide) interdigitated electrode. The electrode width $(w)$ and electrode gap $(g)$ are: $2 / 2,2 / 4,5 / 5,5 / 10$, and 10/10; here $2 / 2$ stands for $w=2 \mu \mathrm{m}$ and $g=2 \mu \mathrm{m}$, etc. The top substrate was a plain glass and the cell gap was controlled at $d \sim 7.5 \mu \mathrm{m}$ by spacer balls. After UV exposure $\left(\lambda \sim 365 \mathrm{~nm}\right.$, intensity $\sim 2 \mathrm{~mW} / \mathrm{cm}^{2}$ for $30 \mathrm{~min}$ ), the polymer/LC composite was stabilized in blue phase over a wide temperature range (from below $0{ }^{\circ} \mathrm{C}$ to $\left.\sim 70{ }^{\circ} \mathrm{C}\right)$. To characterize electro-optic properties, we used a He-Ne laser $(\lambda=633 \mathrm{~nm})$ as probing beam and placed the PS-BPLC sample between two crossed polarizers. To maximize the transmittance, the striped electrode was oriented at $45^{\circ}$ to the transmission axis of the polarizer.

\section{RESULTS AND DISCUSSIONS}

Fig. 1 depicts the measured voltage-dependent transmittance (VT) curves of four PS-BPLC samples with $2 / 4,5 / 5,5 / 10$, and $10 / 10$ electrodes at $25^{\circ} \mathrm{C}$ and $35^{\circ} \mathrm{C}$. The driving signal is 100 $\mathrm{Hz}$ square waves at $50 \%$ duty cycle. Three cycles of ascending and descending voltage scanning from $V=0$ to the peak transmittance voltage $\left(V_{p}\right)$ of each cell were performed consecutively for each sample. To take diffraction effect into consideration, we measured the VT curves under crossed $\left(T_{\perp}\right)$ and parallel polarizers $\left(T_{/ /}\right)$, respectively, and then calculated the normalized transmittance as $T_{\perp}(V) /\left[\mathrm{T}_{\perp}(\mathrm{V})+\mathrm{T}_{/ /}(V)\right][11]$.

In general, the $V_{p}$ of an IPS-based BPLC cell is determined by the Kerr constant and electrode dimension, e.g., electrode width and gap [3]. As shown in Fig. 1, at a given temperature (say $25^{\circ} \mathrm{C}$ ), the measured $V_{p}$ decreases gradually as the electrode width and gap decrease from $5 / 10$ to $5 / 5$, and then to $2 / 4$, with 


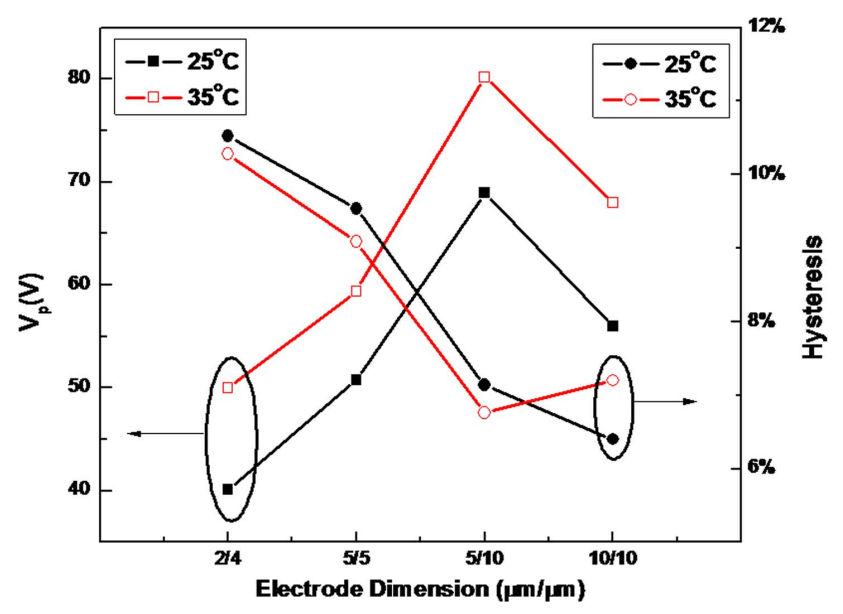

Fig. 2. Measured peak transmittance voltage (left) and hysteresis (right) of the samples shown in Fig. 1.

one exception which is $10 / 10$. This decreasing trend basically agrees with what the UCF model predicts [3], except for the 2/4 structure. Detailed mechanism is related to dielectric coupling as will be explained later.

Fig. 2 shows the measured $V_{p}$ values as denoted by squares. At $25^{\circ} \mathrm{C}$, as the electrode width and gap decrease from $5 / 10$ to $5 / 5$, and then to $2 / 4, V_{p}$ decreases from $70 \mathrm{~V}_{\mathrm{rms}}$ to $50 \mathrm{~V}_{\mathrm{rms}}$ and $40 \mathrm{~V}_{\mathrm{rms}}$, respectively. As for the $10 / 10$ cell, its $V_{p}\left(\sim 55 \mathrm{~V}_{\mathrm{rms}}\right)$ is $\sim 20 \%$ lower than that of $5 / 10$ because of the deeper electric field penetration. However, its transmittance is lower. The contrast ratio (CR) of the 5/5 sample is $\sim 500: 1$.

According to this trend, an even smaller electrode width/gap, such as $2 / 2$, could be helpful for reducing the operating voltage. Thus, we also fabricated such an IPS cell and evaluated its VT curve. However, its phase retardation is small and transmittance is relatively low because the electric field is restricted to the vicinity of electrode. Another serious drawback in hysteresis will be discussed later.

Hysteresis is a general problem in polymer-stabilized LC devices [12]. It affects the accuracy of gray scale control and should be minimized. Also included in Fig. 2 is the measured hysteresis of the four IPS cells studied. Here the hysteresis is defined as the ratio of voltage difference at $50 \%$ of the maximum transmittance $\left(\Delta V_{T 50}\right)$ to $V_{p}$ between the ascending and descending curves. From Fig. 2, the hysteresis gradually increases as the electrode dimension decreases. For the 2/4 IPS cell, the hysteresis reaches $\sim 10.5 \%$. In a BPLC cell, hysteresis is affected by several factors, e.g., polymer type, polymer concentration, UV curing condition, and electric field strength thru electrostriction effect [13]. The latter process has a slow recovery time. For comparison, we took another measurement by driving the sample to $50 \%$ of the peak transmittance and then returning to the dark state gradually. We found the hysteresis is negligible (results are not shown here). This information is very important because most displayed images in a TV are usually in the vicinities of $\sim 50 \%$ gray levels [14], but for desktop monitors the background is usually white.

Next, we measured the dynamic response of the BPLC cells. Fig. 3 presents the measured on-off time of the five samples studied. The samples were operated between $V=0$ and $V_{p}$ states. The rise and decay times were defined as the time difference between $10 \%$ and $90 \%$ of the peak transmittance. The rise

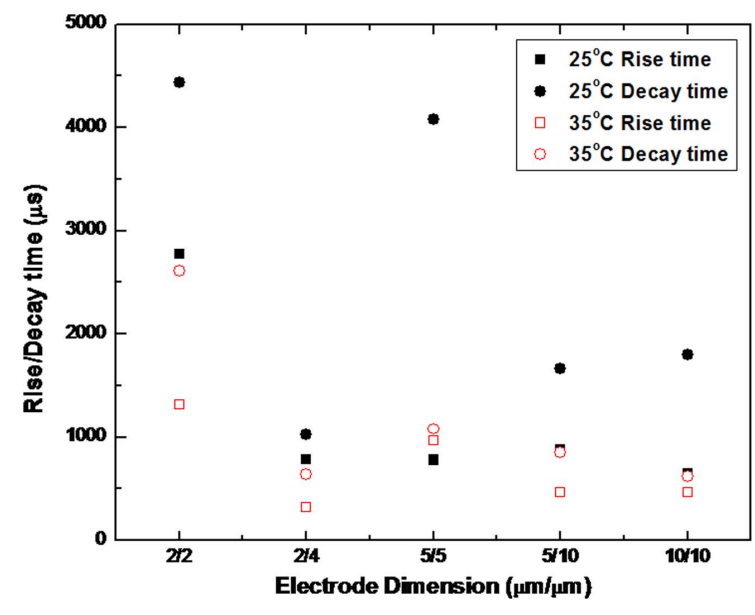

Fig. 3. Measured rise time (squares) and fall time (circles) of the samples with different electrode width/gap by a $100 \mathrm{~Hz}$ square wave driving signal at $25^{\circ} \mathrm{C}$ (solid dots) and $35^{\circ} \mathrm{C}$ (open dots).

time is dependent on the on-state voltage, but decay time is insensitive. Generally speaking, the response time of a polymerstabilized BPLC system is in submillisecond level [5]. However as shown in Fig. 3, the decay time (at $25^{\circ} \mathrm{C}$ ) for the $2 / 4,5 / 10$, and $5 / 10$ devices is below $2 \mathrm{~ms}$, but is slightly over $4 \mathrm{~ms}$ for the $2 / 2$ and $5 / 5$ structures. The decay time of a PS-BPLC depends on the visco-elastic constant $\left(\gamma_{1} / k\right)$ and the pitch length $(p)$ as [15]: $\tau_{\text {off }}=\gamma_{1} p^{2} /\left[(2 \pi)^{2} k_{22}\right]$. Our BPLC mixture JC-BP01M has a very large dielectric anisotropy $(\Delta \varepsilon \sim 94)$; that means it has several polar groups. Thus, its viscosity is fairly large [8]. High viscosity leads to a slower response time. Besides viscosity, from Fig. 3, we found that the electrode dimension also plays an important role affecting the response time. For example, the rise time and decay time of the samples with 1:1 electrode width/gap ratio are longer than those with 1:2 ratio. For the sample with $w=2 \mu \mathrm{m}$ and $g=2 \mu \mathrm{m}$, the total response time is as slow as $\sim 7 \mathrm{~ms}$. This unusual slow response time is believed to arise from electrostriction and phase transition due to strong fields generated by the narrow electrode gap.

Another exciting result shown in Fig. 1 is that the normalized transmittance is over $80 \%$ for all the samples except for the $10 / 10$ structure. These results are higher than those predicted by the UCF model [3]. To understand the responsible mechanisms, we examined our samples under a polarized optical microscope (POM). Fig. 4 shows the photos of three samples: $2 / 2$, $5 / 5$, and $10 / 10$ taken at the room temperature $\left(\sim 23{ }^{\circ} \mathrm{C}\right)$. Before voltage operations, all the samples were stabilized in the blue-phase state. Fig. 4(a) and (b) shows the peak transmittance state of the 5/5 and 10/10 cells, respectively. From Fig. 4(a), we found the $5 / 5$ sample has an unusually larger bright area than the dark region. In a 5/5 device, the ITO width and gap ratio should be 1:1. Therefore, the bright and dark lines should have equal width. From the photos, we estimated the effective aperture ratio for the $5 / 5$ sample is increased from $50 \%$ to $\sim 75 \%$, but it remains $\sim 50 \%$ for the $10 / 10$ sample. This oversized bright region in the $5 / 5$ structure indicates that the liquid crystals at the edges of ITO electrodes also contribute to the overall transmittance, due to the interaction between the liquid crystals at the electrode and gap regions. To explain it in more details, it is considered that liquid crystals at the electrodes region would be affected by the orientation of liquid crystals at the gap region. 

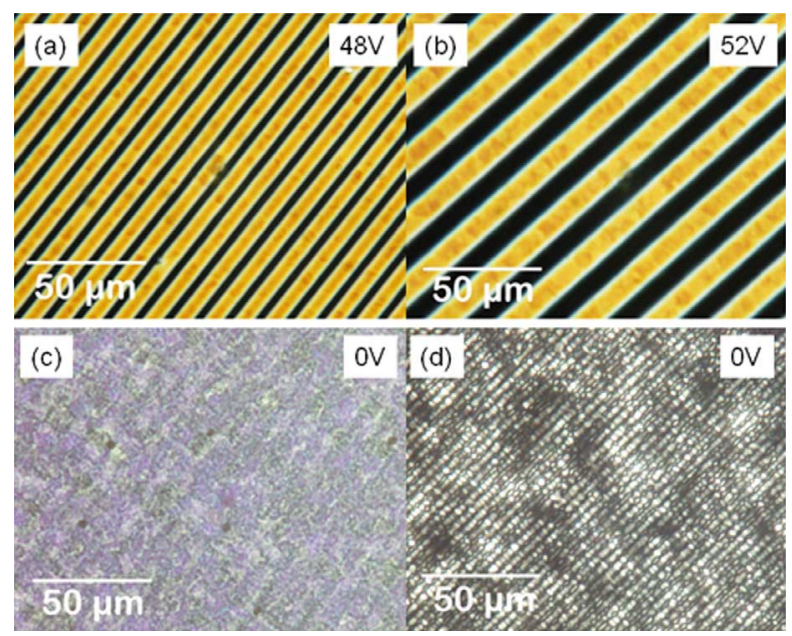

Fig. 4. POM observations of the sample with: (a) 5/5 and (b) 10/10 electrode at the specified peak transmittance voltage; (c) 5/5 and (d) $2 / 2$ electrode at $\mathrm{V}=0$ after voltage operation.

This interaction is more obvious for small electrode dimensions and needs to be taken into consideration. The interaction between liquid crystals is related to the dielectric constants, so we call it dielectric coupling effect. This dielectric coupling effect could also explain why the $2 / 4$ structure has a lower $V_{p}$ than $5 / 5$. However, it is neglected in the UCF model [3] so that the predicted transmittance is lower than that observed experimentally.

To prove that the above-mentioned dielectric coupling effect is more apparent for the large $\Delta \varepsilon \mathrm{LC}$ host, we prepared another BPLC sample with Merck BL-038 $(\Delta n \sim 0.27, \Delta \varepsilon \sim 17)$. Its Kerr constant is $\sim 1 \mathrm{~nm} / \mathrm{V}^{2}$, which is $\sim 10 \mathrm{X}$ smaller than that of JC-BP01M. Similarly, the transmittance of 5/5 structure ( $\sim 60 \%)$ is higher than that of $10 / 10$ structure $(\sim 50 \%)$. However, both transmittances are lower than those using JC-BP01M. Therefore, dielectric coupling effect is weaker for the small $\Delta \varepsilon$ LC host. UCF model is reliable as long as the dielectric couple effect is negligible. For a large $\Delta \varepsilon$ LC like JC-BP01M, the dielectric coupling effect has to be taken into account.

Fig. 4(c) and (d) shows the POM photos of $5 / 5$ and $2 / 2$ samples, respectively, when the voltage was removed instantaneously from their individual $V_{p}$ to 0 . For the $5 / 5$ and $10 / 10$ samples, blue phase state was restored when voltage was switched off. However, by carefully inspecting the 5/5 sample [Fig. 4(c)] we observed white fabric-like patterns. As mentioned before, the electric field in the $5 / 5$ electrode is stronger than that generated in the 10/10 electrode. This strong electric field could cause deformations to the polymer networks and, as a result, lead to white fabric patterns interlaced in the voltage-off state as Fig. 4(c) shows. However in Fig. 4(d), the LC texture did not restore back to the blue phase state; instead, some randomly distributed bright and dark areas are observed. Such a LC texture is very similar to a randomly oriented polymer network liquid crystal. This result again depicted a very strong electric field was induced in the $2 / 2$ electrode like a point discharge effect. Polymer network was damaged and the LC molecules were no longer aligned in the blue phase structure after applying such a strong electric field. The whole system, therefore, transited to other LC phase and the electro-optical characteristics including hysteresis, response time, and contrast ratio were all degraded.

\section{CONCLUSION}

Electrode dimension effects on a large Kerr constant BPLC were evaluated. The operating voltage decreases as the electrode dimension shrinks. With a single-sided IPS electrode with $2-\mu \mathrm{m}$ electrode width and $4-\mu \mathrm{m}$ space, the peak transmittance voltage was reduced to $40 \mathrm{~V}$, while keeping the transmittance over $80 \%$. Under this operating condition, the total response time (rise + decay) is $\sim 2 \mathrm{~ms}$ and hysteresis is $\sim 10 \%$. This large hysteresis is mainly caused by the electrostriction effect due to the strong electric field generated from small electrodes. However, when the electrode width/gap was further reduced to $2 / 2$, the accumulated phase retardation was less than $1 \pi$ due to insufficient penetration depth. Moreover, the electric field generated by the 2/2 electrode is so strong and it could damage the polymer networks and the blue phase structure, resulting in a very slow response time and large hysteresis. Therefore, the electrode dimension cannot be reduced unlimitedly.

\section{ACKNOWLEDGMENT}

The UCF group is indebted to to Dr. Y. Haseba of Chisso for providing the blue phase sample.

\section{REFERENCES}

[1] H. Kikuchi, M. Yokota, Y. Hisakado, H. Yang, and T. kajiyama, "Polymer-stabilized liquid crystal blue phases," Nature Mater., vol. 1, pp. 64-68, Sept. 2002.

[2] Y. Hisakado, H. Kikuchi, T. Nagamura, and T. Kajiyama, "Large electro-optic Kerr effect in polymer-stabilized liquid crystalline blue phases," Adv. Mater., vol. 17, pp. 96-98, Jan. 2005.

[3] Z. Ge, S. Gauza, M. Jiao, H. Xianyu, and S. T. Wu, "Electro-optics of polymer-stabilized blue phase liquid crystal displays," Appl. Phys. Lett., vol. 94, p. 101104, Mar. 2009.

[4] Z. Ge, L. Rao, S. Gauza, and S. T. Wu, "Modeling of blue phase liquid crystal displays," J. Display Technol., vol. 5, no. 7, pp. 250-256, Jul. 2009.

[5] K. M. Chen, S. Gauza, H. Xianyu, and S. T. Wu, "Submillisecond graylevel response time of a polymer-stabilized liquid crystal," J. Display Technol., vol. 6, no. 2, pp. 49-51, Feb. 2010.

[6] L. Rao, Z. Ge, S. T. Wu, and S. H. Lee, "Low voltage blue-phase liquid crystal displays," Appl. Phys. Lett., vol. 95, p. 231101, Dec. 2009.

[7] L. Rao, J. Yan, and S. T. Wu, "Prospects of emerging polymer-stabilized blue-phase liquid crystal displays," J. Soc. Inf. Display, vol. 18, pp. 954-959, Nov. 2010.

[8] L. Rao, J. Yan, S. T. Wu, S. Yamamoto, and Y. Haseba, "A large Kerr constant polymer-stabilized blue phase liquid crystal," Appl. Phys. Lett., vol. 98, p. 081109, Feb. 2011.

[9] S. Yoon, M. Kim, M. S. Kim, B. G. Kang, M. K. Kim, A. K. Srivastava, S. H. Lee, Z. Ge, L. Rao, S. Gauza, and S. T. Wu, "Optimization of electrode structure to improve the electro-optic characteristics of liquid crystal display based on Kerr effect," Liquid Cryst., vol. 37, pp. 201-208, Feb. 2010.

[10] M. Jiao, Y. Li, and S. T. Wu, "Low voltage and high transmittance blue-phase liquid crystal displays with corrugated electrodes," Appl. Phys. Lett., vol. 96, p. 011102, Jan. 2010.

[11] J. Yan, M. Jiao, L. Rao, and S. T. Wu, "Direct measurement of electricfield-induced birefringence in a polymer-stabilized blue-phase liquid crystal composite," Opt. Express, vol. 18, pp. 11450-11455, May 2010.

[12] K. M. Chen, S. Gauza, H. Xianyu, and S. T. Wu, "Hysteresis effects in blue-phase liquid crystals," J. Display Technol., vol. 6, no. 8, pp. 318-322, Aug. 2010.

[13] Y. Haseba, H. Kikuchi, T. Nagamura, and T. Kajiyama, "Large electrooptic Kerr effect in nanostructured chiral liquid-crystal composites over a wide temperature range," Adv. Mater., vol. 17, pp. 2311-2315, Oct. 2005.

[14] H. C. Cheng, I. B. David, and S. T. Wu, "Five-primary-color LCDs," J. Display Technol., vol. 6, no. 1, pp. 3-7, Jan. 2010.

[15] H. F. Gleeson and H. J. Coles, "Dynamic properties of blue-phase mixtures," Liquid Cryst., vol. 5, pp. 917-926, 1989. 